\title{
Correlative Microscopy-Novel Methods and Their Applications to Explore 3D Chemistry and Structure of Nanoscale Lattice Defects: A Case Study in Superalloys
}

\author{
S.K. MAKINENI ${ }^{1,4}$ M. LENZ, ${ }^{2}$ P. KONTIS,${ }^{1}$ Z. LI, ${ }^{1}$ A. KUMAR,${ }^{1}$ \\ P.J. FELFER ${ }^{3}$ S. NEUMEIER, ${ }^{3}$ M. HERBIG,${ }^{1}$ E. SPIECKER ${ }^{2}$ \\ D. RAABE ${ }^{1,5}$ and B. GAULT (iD ${ }^{1,6}$ \\ 1.-Max-Planck-Institut für Eisenforschung GmbH, 40237 Düsseldorf, Germany. 2.-Institute \\ of Micro- and Nanostructure Research and Center for Nanoanalysis and Electron Microscopy \\ (CENEM), Friedrich-Alexander-Universität Erlangen-Nürnberg, Cauerstraße 6, 91058 Erlangen, \\ Germany. 3.-Department of Materials Science, Institute for General Materials Properties, Friedrich- \\ Alexander University Erlangen-Nürnberg, Erlangen, Germany. 4.-e-mail: sk.makineni@mpie.de. \\ 5.—e-mail: d.raabe@mpie.de. 6.—e-mail: b.gault@mpie.de
}

\begin{abstract}
Nanoscale solute segregation to or near lattice defects is a coupled diffusion and trapping phenomenon that occurs in superalloys at high temperatures during service. Understanding the mechanisms underpinning this crucial process will open pathways to tuning the alloy composition for improving the high-temperature performance and lifetime. Here, we introduce an approach combining atom probe tomography with high-end scanning electron microscopy techniques, in transmission and backscattering modes, to enable direct investigation of solute segregation to defects generated during high-temperature deformation such as dislocations in a heat-treated Ni-based superalloy and planar faults in a CoNi-based superalloy. Three protocols were elaborated to capture the complete structural and compositional nature of the targeted defect in the alloy.
\end{abstract}

\section{INTRODUCTION}

Correlative microscopy, i.e., the combined utilization of a range of microscopy techniques on a single specimen, is increasingly deployed to understand fundamental aspects in material science. The combination of transmission electron microscopy (TEM) and atom probe tomography (APT) enables researchers to relate the atomic structure and composition of nanoscale features of interest and has been gaining influence over the past decades. Typically, correlative TEM/APT analyses have been carried out from distinct specimens from the same alloy, ${ }^{1-7}$ which fails in cases where the microstructure is inhomogeneous and/or when targeting rare microstructural features for both TEM and APT investigations. As recently reviewed by Herbig, ${ }^{8}$ full electron microscopy characterization of specific features can be carried out on needle-shaped specimens prior to APT analysis by utilizing specially designed holders. This approach has, for example, led to a better understanding of diffusional mechanisms resulting in local solute segregation at crystalline imperfections ${ }^{9-14}$ that critically impact the macroscopic material behavior. APT had previously revealed details of the composition of structural imperfections,${ }^{15}$ the presence of which were confirmed by field ion microscopy ${ }^{16}$ or TEM. ${ }^{11,17,18}$ Here, we introduce methodologies to aid target features of interest for correlative TEM/APT investigation by exploiting advanced scanning electron microscopy (SEM) techniques in deformed Ni- and CoNi-based superalloys, one of the most important classes of engineering materials for temperatures above $1000^{\circ} \mathrm{C}$. Their high temperature stability is attributed to the uniform distribution of $\mathrm{L} 1_{2}$-ordered $\gamma^{\prime}$ precipitates coherently embedded in an fcc solid solution $\gamma$ matrix. Three protocols for site-specific correlative investigation are presented.

\section{MATERIALS AND METHODS}

\section{Materials}

The polycrystalline Ni-based superalloy IN792, commonly used in land-based gas turbines, was studied. Its chemical composition is $\mathrm{Ni}-13.9 \mathrm{Cr}-$ 
8.8Co-1.1Mo-1.3W-7.6Al-4.9Ti-1.3Ta-0.4C-0.1B$0.012 \mathrm{Zr}$ (at.\%). It was provided as 20-mm-diameter bars by Howmet. A hot isostatic press (HIP) process at $1195^{\circ} \mathrm{C}$ and $150 \mathrm{MPa}$ for $2 \mathrm{~h}$ was followed by a solution heat treatment stage at $1121^{\circ} \mathrm{C}$ for $2 \mathrm{~h}$ and a final stage of aging at $850^{\circ} \mathrm{C}$ for $24 \mathrm{~h}$. After heat treatment, specimens were ground and polished with abrasive media to a $1-\mu \mathrm{m}$ finish and were isothermally exposed at $750^{\circ} \mathrm{C}$ for $50 \mathrm{~h}$.

A single-crystal CoNi-based superalloy with the composition $\mathrm{Co}-32 \mathrm{Ni}-8 \mathrm{Al}-5 \mathrm{~W}-6 \mathrm{Cr}-2.5 \mathrm{Ti}-1.5 \mathrm{Ta}-$ $0.1 \mathrm{Hf}-0.4 \mathrm{Si}$ (at.\%) was prepared by the Bridgman process. The heat treatment steps were $1280^{\circ} \mathrm{C} /$ $8 \mathrm{~h}+1050^{\circ} \mathrm{C} / 5 \mathrm{~h}+900^{\circ} \mathrm{C} / 16 \mathrm{~h}$ to obtain a uniform $\gamma / \gamma^{\prime}$ microstructure. Tensile specimens were cut and crept at $850^{\circ} \mathrm{C}$ with an applied stress of $400 \mathrm{MPa}$ along the [001]-direction up to $4.6 \%(380 \mathrm{~h})$ and $0.3 \%$ (40 h) plastic strain. Cross sections close to the $\{100\}$ and $\{110\}$ habit planes were cut and mechanically polished for further microstructural characterization.

\section{Controlled Electron Channeling Contrast Imaging (cECCI)}

A Zeiss Merlin scanning electron microscope (Carl Zeiss SMT AG, Germany) with a Gemini-type field emission gun electron column and a Bruker e-Flash HR EBSD detector (Bruker Corp., USA) was used. ECCI (electron channeling contrast imaging) under controlled diffraction conditions was employed to rotate and tilt the crystal into two-beam diffraction conditions using the TOCA (Tools for Orientation Determination and Crystallographic Analysis computer program; for details, see Zaefferer et al. ${ }^{19}$ ). The microscope was operated at $30 \mathrm{kV}$ accelerating voltage and 3-4 nA probe current.

\section{Focused Ion Beam (FIB) Preparation}

Atom probe specimens were fabricated using a dual-beam SEM/focused-ion-beam (FIB) instrument (FEI Helios Nanolab 600 and 660 equipped with a STEM detector). The specimens were sharpened by FIB milling followed by a final cleaning procedure at $2 \mathrm{kV}$ and 16-pA current to remove severely damaged regions induced by the previously employed high-energy $(30 \mathrm{kV}) \mathrm{Ga}$ ion beam.

\section{Transmission Electron Microscopy (TEM)}

Transmission electron microscopy (TEM) on the APT specimen was carried out using a Phillips CM20 operating at $200 \mathrm{kV}$. High-resolution imaging was carried out in STEM mode using an aberration corrected Titan FEI microscope operated at $300 \mathrm{kV}$.

\section{Atom Probe Tomography (APT)}

APT measurements were conducted using instruments from Cameca Instruments Inc.: a LEAP 5000XR, LEAP 5000XS and LEAP 4000 HR. The laser pulsing mode was applied at a pulse repetition rate between 100 and $250 \mathrm{kHz}$ and a pulse energy between 30 and 40 pJ. The specimen's base temperature was kept at $40 \mathrm{~K}$, and the target detection rate was set to be 5 ions detected every 1000 pulses on average. Data analysis was performed using the IVAS 3.6.14 software package.

\section{RESULTS AND DISCUSSION}

\section{Method (1) cECCI-Guided Conventional Tar- get Preparation}

First, ECC imaging is used to locate a region of interest (ROI) with high dislocation density in the IN792 alloy, generated during the volume expansion of oxidized carbide in the $\gamma / \gamma^{\prime}$ microstructure as shown in Fig. 1a. ${ }^{20,21}$ A series of APT specimens were prepared from locations highlighted in blue following the conventional procedure outlined by Thompson et al. ${ }^{22}$ and using a commercially available flat-top Si coupon and conventional clip holder. A representative tomographic reconstruction is shown in Fig. 1b: two $\gamma^{\prime}$ precipitates and their interfaces with the $\gamma$ matrix are imaged. Within $\gamma^{\prime}$, a $\mathrm{Cr}$ isoconcentration surface (3 at.\%) reveals $\gamma$ particles that form during the $750^{\circ} \mathrm{C}$ heat treatment, ${ }^{23,24}$ while the tubular features correspond to solute segregation to dislocations. A linear compositional profile plotted from a cylindrical ROI perpendicular to the dislocation, along the arrow labeled \#1, plotted in Fig. 1c, reveals clear segregation of $\mathrm{Cr}$ and $\mathrm{Co}$ to the dislocations, which contrasts with a depletion in $\mathrm{Ni}, \mathrm{Al}$ and $\mathrm{Ti}$.

\section{Method (2) cECCI-Guided In-plane Target Preparation for TEM and APT}

Here again, ECC imaging is performed to reveal and locate lattice defects within the crept $\mathrm{CoNi}$ based superalloy as summarized in Fig. 2a-b. Figure $2 \mathrm{a}$ is obtained by controlled rotation/tilting of the sample such that its surface normal is close to the [110] direction of the fcc-crystalline lattice and with the $g=(00 \overline{2})$ two-beam condition, which shows a clear atomic density contrast between the dark fcc- $\gamma$ matrix and the bright $\mathrm{L} 1_{2}-\gamma^{\prime}$ precipitates rafted along the creep-loading direction. High-number density of stacking faults (SFs) appears as bright features with oscillating intensity. These are visible because of shearing of $\gamma^{\prime}$, which is the primary active deformation mechanism operating during high temperature creep in this type of alloy. ${ }^{25}$ From the stereographic projection in Fig. $2 \mathrm{~b}$, aligned according to the sample orientation, four variants of SFs are observed, marked as A, B, C and $\mathrm{D}$ according to their traces on the $\{111\}$ planes. SF variants $A$ and $D$ are associated with the $(11 \overline{1})$ and (111) planes forming angles of $38^{\circ}$ and $36^{\circ}$ with the sample surface and exhibit fading oscillating contrast. This fading contrast arises because of the loss of backscattered signal from the inclined stacking faults with increasing depth below the sample 


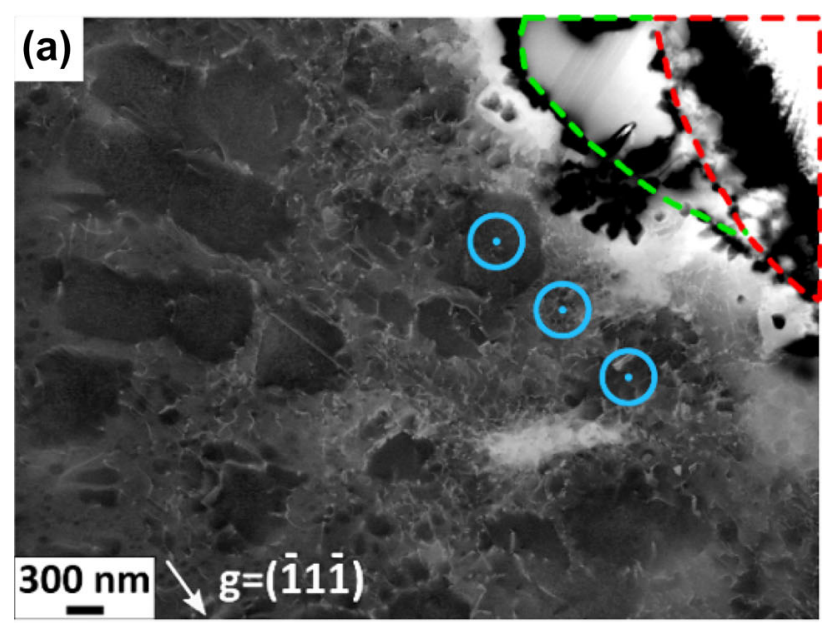

(b)

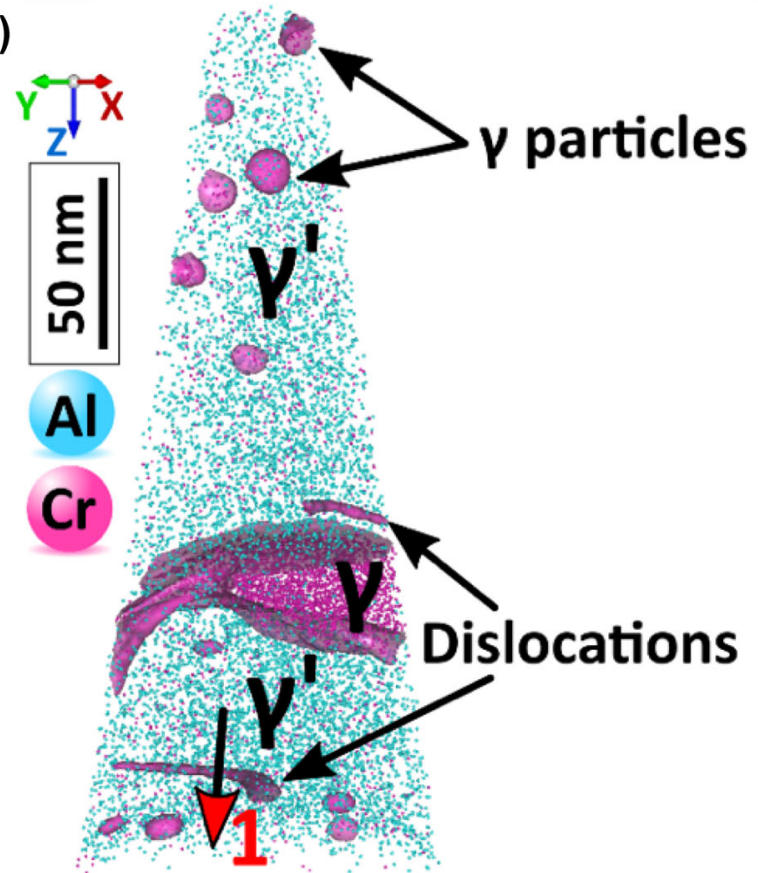

(c)

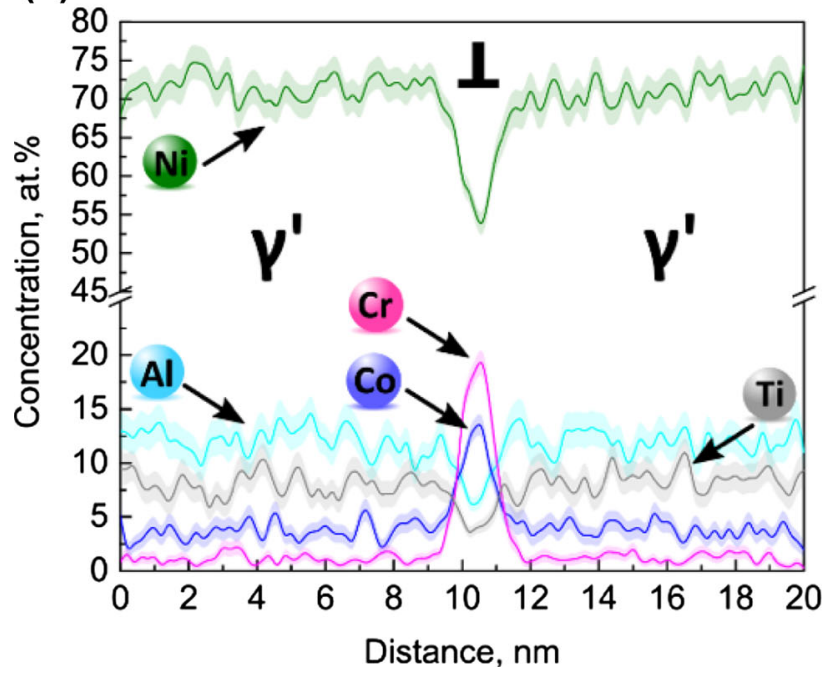

4Fig. 1. Method (1): (a) CECCI micrograph from the cross section of an oxidized intragranular MC carbide in the IN792 alloy after isothermal exposure at $750^{\circ} \mathrm{C}$ for $50 \mathrm{~h}$. Part of the oxidized $\mathrm{MC}$ carbide is denoted by the red dashed line, and the recrystallized zone is denoted by the green dashed line. (b) Atom probe reconstruction from a region with high dislocation density next to an oxidized MC carbide showing $\gamma / \gamma^{\prime}$ interfaces, dislocations, and $\gamma$ particles within the $\gamma^{\prime}$ precipitate. (c) 1D concentration profile perpendicular to the denoted dislocation in (b) (arrow \#1) (Color figure online).

surface. The $\mathrm{B}$ and $\mathrm{C}$ variants appear near edge-on belonging to the $(\overline{1} 11)$ and $(\overline{1} 1 \overline{1})$ planes forming angles of $80^{\circ}$ and $81^{\circ}$ with the sample surface and thus appear as sharp bright lines without fading intensity of the oscillating contrast.

Once an ROI, highlighted in yellow, is identified in the bulk sample as shown in Fig. 2c and located in the FIB as indicated by the black box in Fig. 2d, it is marked and protected by in situ Pt deposition; see Fig. 2d-e. Figure $2 \mathrm{f}-\mathrm{k}$ shows the step-by-step inplane lift-out 26,27 procedure schematically, where cut parts of the lift-out are positioned/attached on the electro-polished tips of a halved TEM Mo grid, which is held in a dedicated holder ${ }^{8,28}$ and subsequently shaped into conical APT specimens by annular milling. ${ }^{29}$

Figure 3a shows a bright-field TEM image of the APT specimen containing a C-type SF close to the [110] zone axis and in a $g=(00 \overline{2})$ two-beam condition. A straight line appears with a contrast resulting from the intersection of the staking fault with the surface of the APT specimen. Figure $3 \mathrm{~b}$ shows a HAADF-STEM image of the APT tip close to the [110] zone axis. The bright contrast, with respect to the surrounding lattice, along the SF indicates segregation of heavy elements. ${ }^{30,31}$ Figure $3 \mathrm{c}$ shows a high-resolution STEM image revealing the $\mathrm{D} 0_{19}$ ordered structure of the fault plane surrounded by the $\mathrm{L} 1_{2} \gamma^{\prime}$ precipitate. ${ }^{31,32}$ A missing A-type plane as visible in Fig. 3d reveals that this is an intrinsic SF. Figure $3 \mathrm{e}-\mathrm{g}$ shows the corresponding APT reconstructions, which has the overall composition of $\gamma^{\prime}$. Figure $3 \mathrm{e}-\mathrm{f}$ depicts the distribution of $\mathrm{Al}$ atoms (brown) and W atoms (red), respectively. Towards the bottom of the tomogram, a planar depletion of $\mathrm{Al}$ contrasting with an enrichment in W becomes apparent. Figure $3 \mathrm{~g}$ displays the $\mathrm{Al}$ compositional map plotted and projected along the y direction onto the xz plane of a cuboidal ROI, which highlights the local Al deficiency at the SF. Figure $3 \mathrm{~h}$ shows the concentration profiles across the fault plane indicating $\mathrm{Co} / \mathrm{W}$ enrichment and $\mathrm{Ni} / \mathrm{Al}$ depletion. Similar compositional segregation effects in the stacking faults have been reported even in crept Co-based superalloys. ${ }^{32}$ 
Correlative Microscopy-Novel Methods and Their Applications to Explore 3D Chemistry and Structure of Nanoscale Lattice Defects: A Case Study in Superalloys

(a)
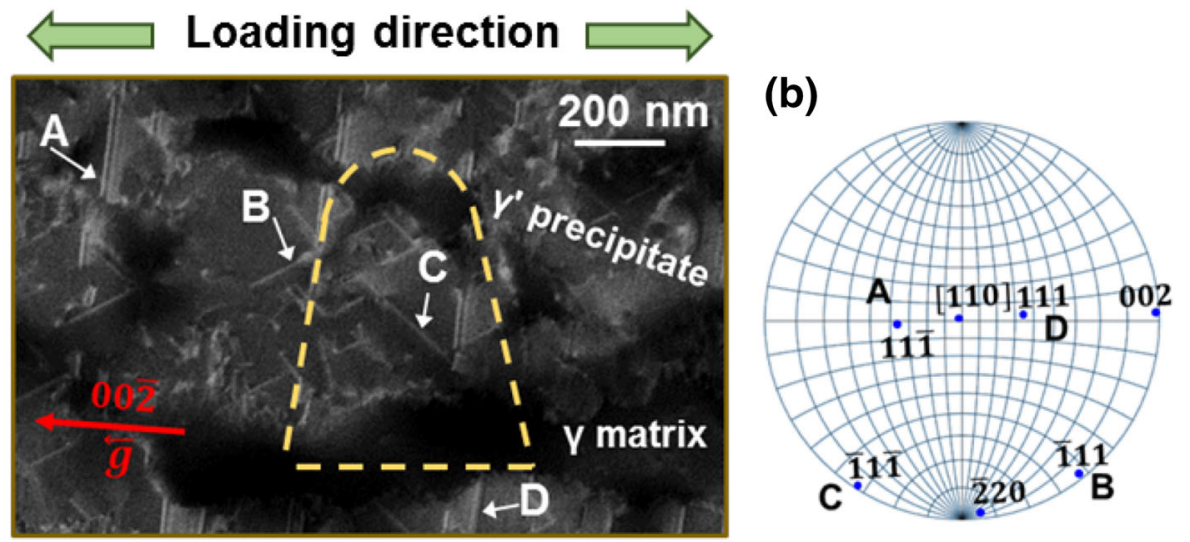

(c)

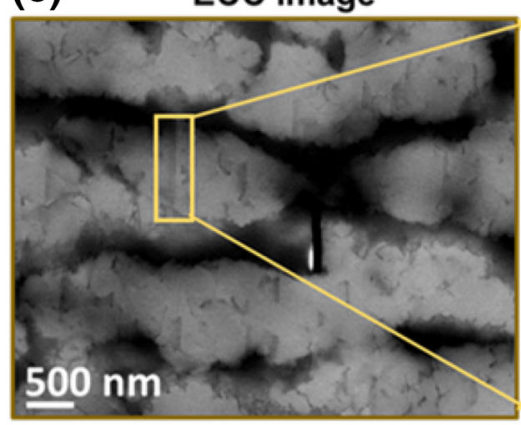

(d)
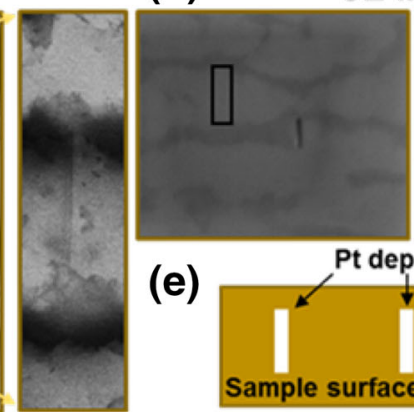

SE image (f)

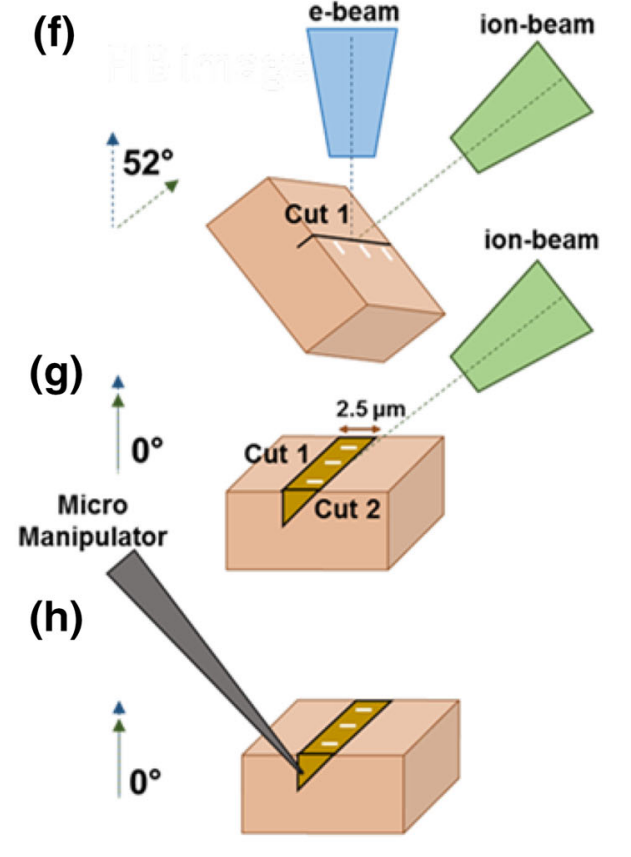

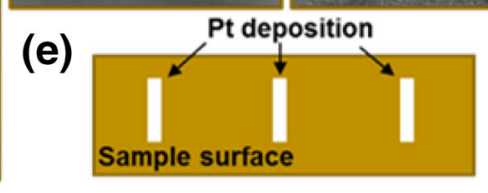

(i)
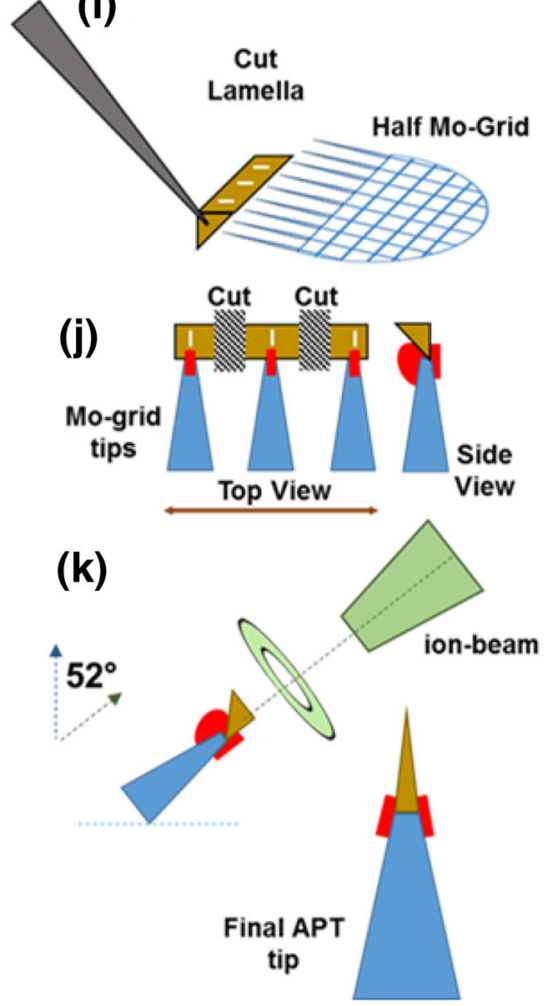

Fig. 2. Method (2): (a) cECC imaging of the creep-deformed CoNi-based superalloy with the sample surface normal close to the [110] zone axis revealing numerous SFs and (b) corresponding stereographic projection. (c) Locating the defect feature in the bulk sample by ECC imaging. (d, e) Marking the feature with Pt deposition (white color) in FIB/SEM. ( $f-k)$ Steps involving the in-plane lift-out procedure to prepare the defect of interest into the apex of an APT tip to conduct correlative microscopy. 
(a)

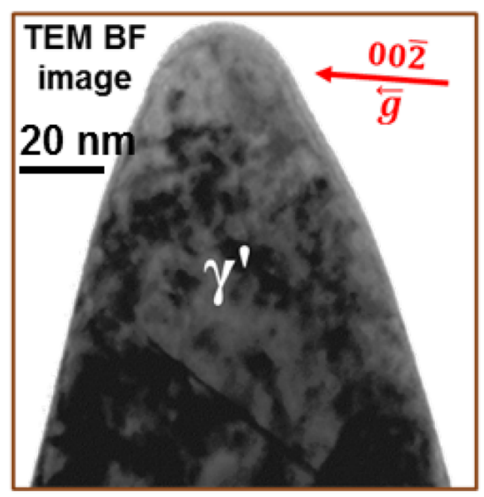

(e)

(e)

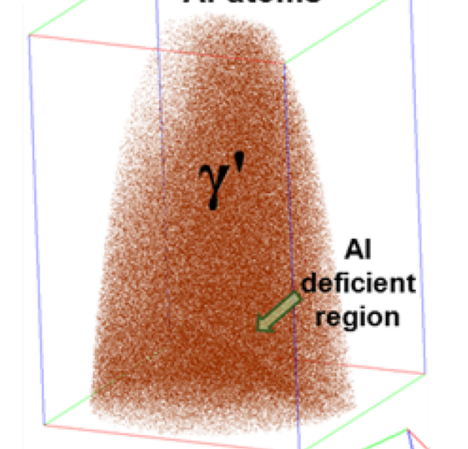

(b)

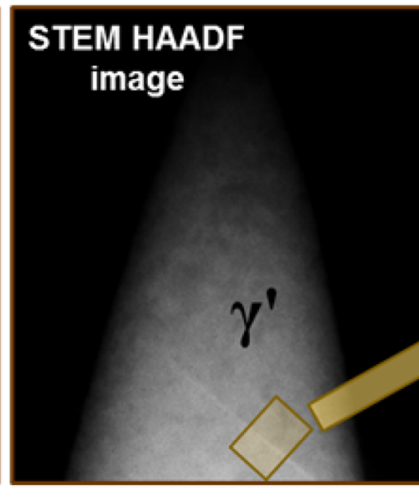

(f)

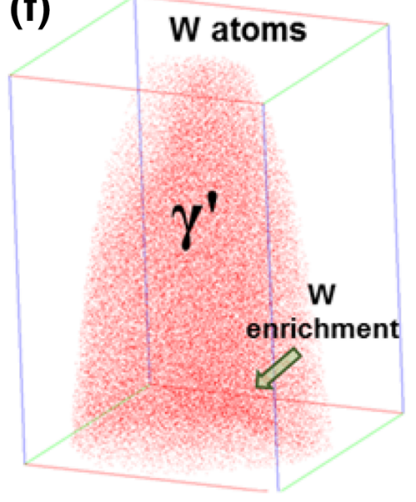

(c)

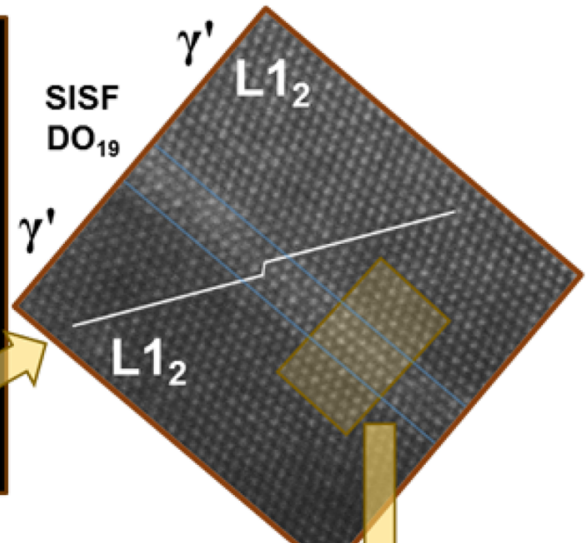

(d)

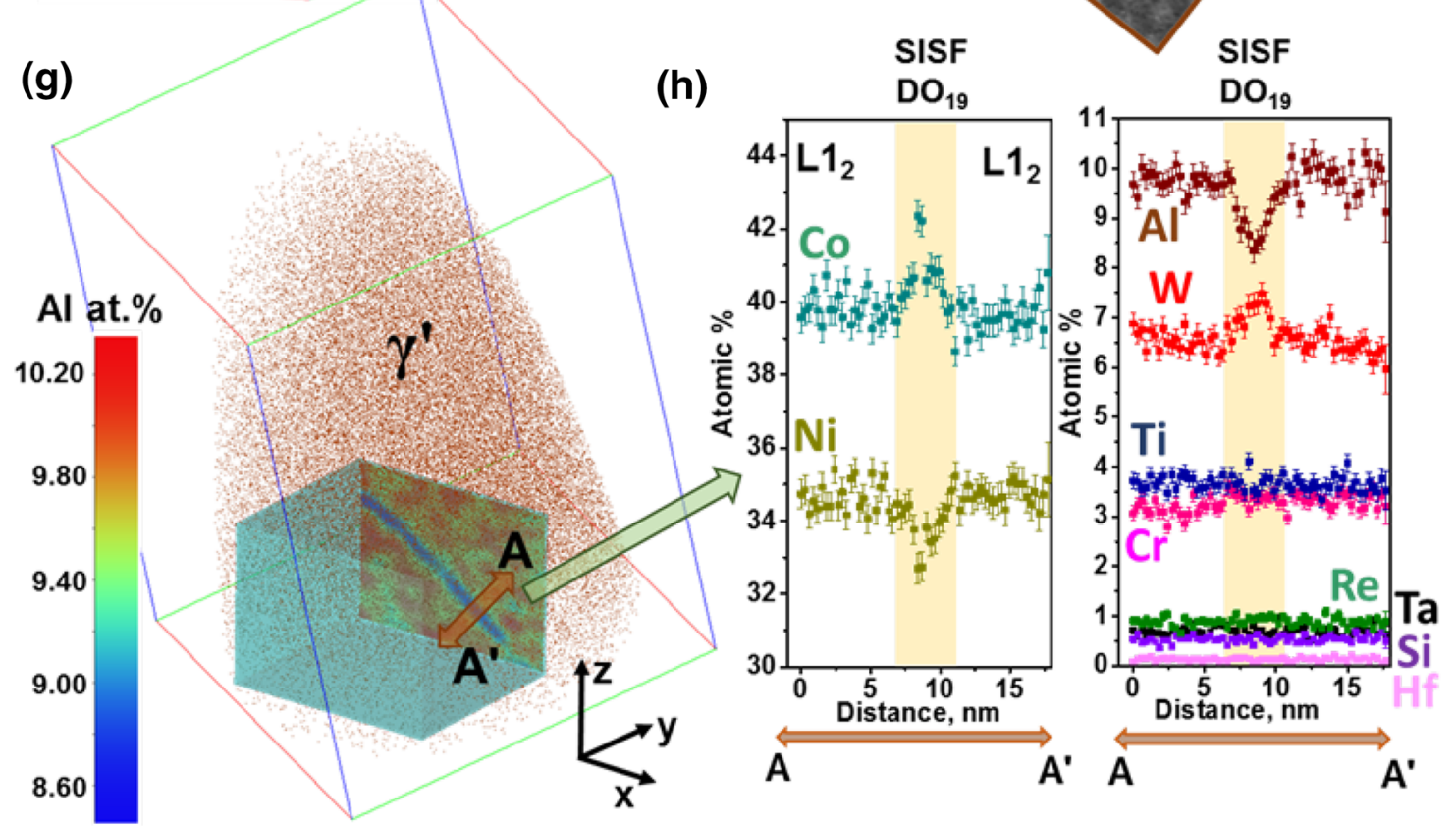

Fig. 3. Method (2): (a) Bright field (BF) TEM image of a $\gamma^{\prime}$ precipitate taken near to the [110] zone axis. An SF is present, appearing as a dark contrast line. (b) HAADF STEM image showing bright $Z$ contrast along the fault plane, indicating segregation of higher $Z$ solutes. (c, d) Highresolution STEM image of the atomic structure of the SF viewed edge-on along the [110] direction. (e-h) APT reconstructed tomogram showing Al deficiency and $\mathrm{W}$ enrichment along the fault plane. 

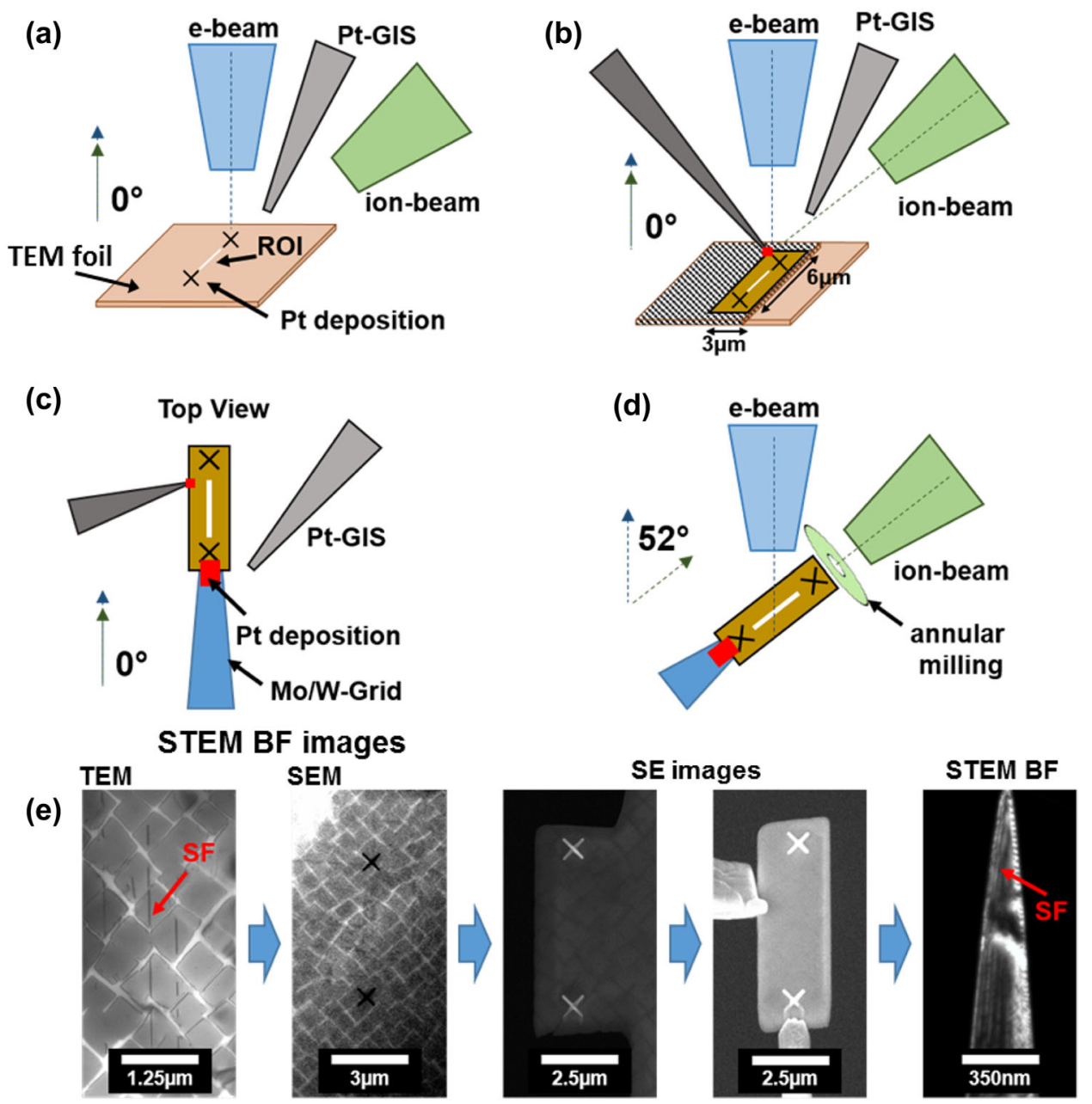

Fig. 4. Method (3): (a) Marking of the ROI with Pt deposition or C contamination in a TEM foil. (b-d) Successive steps of the specimen preparation from a TEM foil (pink color); (e) corresponding series of electron micrographs obtained in the SEM and TEM for the final tip to carry out APT analysis (Color figure online).

\section{Method (3): TSEM-Guided Target Preparation from TEM Foil for APT}

A third method offers the possibility to prepare APT specimens directly from pre-thinned TEM foils. Method (3) allows a detailed TEM characterization on a larger area and the selection and target preparation of a sparsely distributed defect type from this lamella into the APT specimen tip. Figure 4 shows the schematic step-wise preparation procedure for the case of an SF ribbon in the $0.3 \%$ crept CoNi-based superalloy.

Here, the ROI does not show any variation in Zcontrast, and the SFs are not detectable with SE (secondary electron) or BSE (back-scattered electron) detectors. The ROI is identified in the STEM mode in the SEM and marked with Pt or C X-shaped patterns, as shown in Fig. 4a.

In cases where TSEM imaging is not available, a convergent beam focused on the sample's surface can be used to mark the ROI by carbon

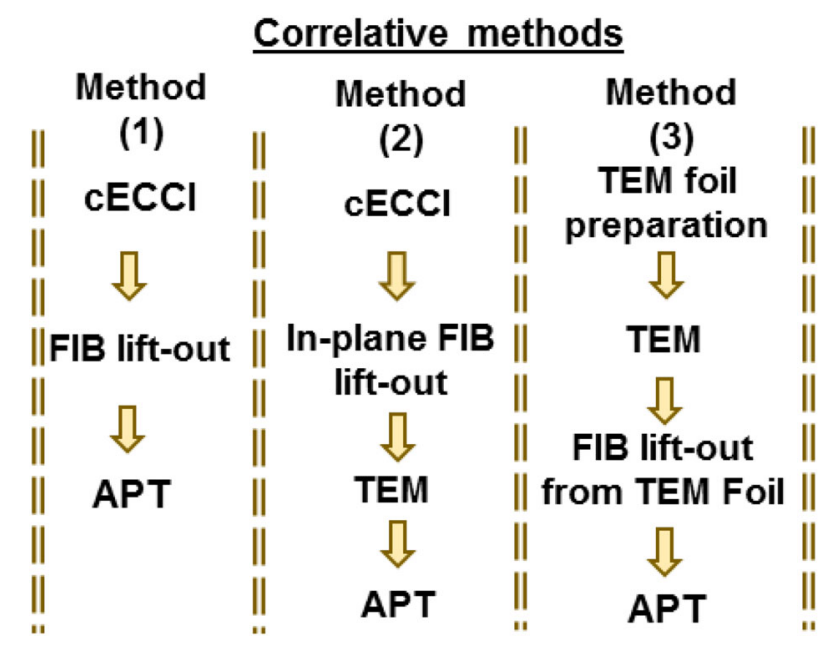

Fig. 5. Sequence of experimental steps in the three methods adopted for the correlative microscopy. 
contamination in the TEM. A $3 \mu \mathrm{m} \times 6 \mu \mathrm{m}$ lamella is cut out at $15 \mathrm{kV}$ and attached to the micromanipulator using Pt deposition; see Fig. 4b. Figure 4c shows the lamella transferred and welded on both sides to a pre-sharpened Mo or $\mathrm{W}$ grid. The lamella must be parallel to the supporting needle, and the dimensions of the stump should not exceed 1-2 $\mu \mathrm{m}$. Finally, as depicted in Fig. 4d, annular milling is performed, with a last milling step at $2 \mathrm{kV}$ with an inner pattern diameter of approximately $50 \mathrm{~nm}$. The X-shaped fiducials enable estimation of the milling depth and position of the ROI. Figure 4e exemplifies the preparation procedure from defect identification and marking to a final APT specimen containing an SF parallel to the specimen's main axis. The preparation is carried out continuously without transfer or breaking of the vacuum and is hence suitable for materials prone to oxidation. In this approach, the ROI is not milled out of bulk material but merely cut out of an already thinned area. This allows for short times under ion radiation when using $15-30 \mathrm{kV}$ for cutting. Beam-sensitive materials can also be processed with voltages down to $5 \mathrm{kV}$ in reasonable times. Shorter radiation times and/or lower acceleration voltages both result in lower overall ion beam damage in the ROI. In addition, the presented method only requires a small sample volume from the TEM foil and thus the foil can be reused for further TEM structural characterization.

This method is suitable for foil thicknesses in the range of 50-250 $\mathrm{nm}$. Foils thinner than typical APT specimen dimensions will result in asymmetric tips and an enhanced risk for severe ion beam damage. TSEM and/or TEM investigations beforehand allow the identification of features of interest in thicker regions of the specimen and an assessment of their representativeness. Advantages of low-energy TSEM have been discussed in detail by Callahan et al. ${ }^{33}$ In the case of the example in Fig. 4, the fault ribbon was traced from the hole in the TEM foil back to a thicker region to avoid the aforementioned issues. No specimens with a thickness $<80 \mathrm{~nm}$ were prepared but we would recommend ion beam energies below $5 \mathrm{kV}$ and "blind" cutting without the acquisition of ion images during the process. Please note that APT specimens from thin TEM foils will exhibit smaller dimensions, and the potential for ion damage in the specimen is increased.

\section{SUMMARY AND CONCLUSION}

Figure 5 summarizes the three experimental protocols introduced herein to characterize atomicscale segregation at dislocations in a crept polycrystalline Ni-based superalloy and at SFs in a crept CoNi-based single-crystal alloy. These nanoscale defects critically impact the mechanical performance of high-temperature engineering alloys. These three protocols are also applicable to any other material or defect features. The strength of these approaches is to enable both structural and compositional information on the same object, which is necessary to unveil the mechanisms controlling the mechanical response of the material under investigation.

\section{ACKNOWLEDGEMENTS}

Open access funding provided by Max Planck Society. The authors are grateful to U. Tezins and A. Sturm for their technical support of the atom probe tomography and focus ion beam facilities at the Max-Planck-Institut für Eisenforschung. SKM acknowledges financial support from the Alexander von Humboldt Foundation. The authors also acknowledge financial support from the DFG SFB TR 103 through projects A1, A4, A7 and B3.

\section{OPEN ACCESS}

This article is distributed under the terms of the Creative Commons Attribution 4.0 International License (http://creativecommons.org/licenses/by/4.0/ ), which permits unrestricted use, distribution, and reproduction in any medium, provided you give appropriate credit to the original author(s) and the source, provide a link to the Creative Commons license, and indicate if changes were made.

\section{REFERENCES}

1. Y.J. Li, P. Choi, C. Borchers, S. Westerkamp, S. Goto, D. Raabe, and R. Kirchheim, Acta Mater. 59, 3965 (2011).

2. C. Fuller, J. Murray, and D. Seidmann, Acta Mater. 53, 5401 (2005).

3. S. Nag, A. Devaraj, R. Srinivasan, R.E.A. Williams, N. Gupta, G.B. Viswanathan, J.S. Tiley, S. Banerjee, S.G. Srinivasan, H.L. Fraser, and R. Banerjee, Phys. Rev. Lett. 106, 245701 (2011).

4. B. Gault, F. de Geuser, L. Bourgeois, B.M.M. Gabble, S.P.P. Ringer, and B.C.C. Muddle, Ultramicroscopy 111, 683 (2011).

5. S.K. Makineni, S. Sugathan, S. Meher, R. Banerjee, S. Bhattacharya, S. Kumar, and K. Chattopadhyay, Sci. Rep. 7, 11154 (2017).

6. M. Dumont, W. Lefebvre, B. Doisneau-Cottignies, and A. Deschamps, Acta Mater. 53, 2881 (2005).

7. R.K.W. Marceau, G. Sha, R. Ferragut, A. Dupasquier, and S.P. Ringer, Acta Mater. 58, 4923 (2010).

8. M. Herbig, Scr. Mater. 148, 98 (2017).

9. M. Herbig, D. Raabe, Y.J. Li, P. Choi, S. Zaefferer, and S. Goto, Phys. Rev. Lett. 112, 126103 (2014).

10. A. Kwiatkowski da Silva, G. Leyson, M. Kuzmina, D. Ponge, M. Herbig, S. Sandlöbes, B. Gault, J. Neugebauer, and D. Raabe, Acta Mater. 124, 305 (2017).

11. M. Kuzmina, M. Herbig, D. Ponge, S. Sandlobes, and D. Raabe, Science 349, 1080 (2015).

12. M.J. Yao, E. Welsch, D. Ponge, S.M.H. Haghighat, S Sandlöbes, P. Choi, M. Herbig, I. Bleskov, T. Hickel, M. Lipinska-Chwalek, P. Shanthraj, C. Scheu, S. Zaefferer, B. Gault, and D. Raabe, Acta Mater. 140, 258 (2017).

13. B.P. Gorman, D.R. Diercks, N. Salmon, E.A. Stach, G. Amador, and C. Hartfield, Microsc. Today 16, 42 (2008).

14. D. Diercks, B.P. Gorman, C.L. Cheung, and G. Wang, Microsc. Microanal. 15, 254 (2009).

15. D. Blavette, E. Cadel, A. Fraczkeiwicz, and A. Menand, Science 286, 2317 (1999).

16. J. Wilde, A. Cerezo, and G.D. Smith, Scr. Mater. 43, 39 (2000). 
17. B. Gault, M.P. Moody, J.M. Cairney, and S.P. Ringer, Mater. Today 15, 378 (2012).

18. G.D.W. Smith, D. Hudson, P.D. Styman, and C.A. Williams, Philos. Mag. 93, 3726 (2013).

19. S. Zaefferer and N.-N. Elhami, Acta Mater. 75, 20 (2014).

20. P. Kontis, Z. Li, D.M. Collins, J. Cormier, D. Raabe, and B. Gault, Scr. Mater. 145, 76 (2018).

21. P. Kontis, D.M. Collins, A.J. Wilkinson, R.C. Reed, D. Raabe, and B. Gault, Scr. Mater. 147, 59 (2018).

22. K. Thompson, D. Lawrence, D.J. Larson, J.D. Olson, T.F. Kelly, and B. Gorman, Ultramicroscopy 107, 131 (2007).

23. M. Segersäll, P. Kontis, S. Pedrazzini, P.A.J. Bagot, M.P. Moody, J.J. Moverare, and R.C. Reed, Acta Mater. 95, 456 (2015).

24. V. Yardley, I. Povstugar, P.-P. Choi, D. Raabe, A.B. Parsa, A. Kostka, C. Somsen, A. Dlouhy, K. Neuking, E.P. George, and G. Eggeler, Adv. Eng. Mater. 18, 1556 (2016).

25. F. Xue, C.H. Zenk, L.P. Freund, M. Hoelzel, S. Neumeier, and M. Göken, Scr. Mater. 142, 129 (2018).
26. P.J. Felfer, T. Alam, S.P. Ringer, and J.M. Cairney, Microsc. Res. Tech. 75, 484 (2012).

27. A. Stoffers, J. Barthel, C.H. Liebscher, B. Gault, O. Cojocaru-Mirédin, C. Scheu, and D. Raabe, Microsc. Microanal. 23, 291 (2017).

28. M. Herbig, P. Choi, and D. Raabe, Ultramicroscopy 153, 32 (2015).

29. D.J. Larson, D.T. Foord, A.K. Petford-Long, H. Liew, M. Blamire, A. Cerezo, and G.D.W. Smith, Ultramicroscopy 79, 287 (1999).

30. M.S. Titus, A. Mottura, G. Babu Viswanathan, A. Suzuki, M.J. Mills, and T.M. Pollock, Acta Mater. 89, 423 (2015).

31. Y. Eggeler, J. Müller, M.S. Titus, A. Suzuki, T.M. Pollock, and E. Spiecker, Acta Mater. 113, 335 (2016).

32. M.S. Titus, R.K. Rhein, P.B. Wells, P.C. Dodge, G.B. Viswanathan, M.J. Mills, A.V. der Ven, and T.M. Pollock, Sci. Adv. 2, e1601796 (2016).

33. P.G. Callahan, J.-C. Stinville, E.R. Yao, M.P. Echlin, M.S. Titus, M. De Graef, D.S. Gianola, and T.M. Pollock, Ultramicroscopy 186, 49 (2018). 